\title{
Study on a Composite Fiberboard with Multiple Electromagnetic Shielding Effectiveness
}

\author{
Chuwang Su ${ }^{*}$, , Quanping Yuan ${ }^{1}$, Weixing Gan ${ }^{1}$, Dawang Dai ${ }^{2}$, Jingda Huang ${ }^{1}$ and Yuanyi Huang ${ }^{1}$ \\ ${ }^{I}$ Forestry College of Guangxi University, Nanning, Guangxi, 530004, China \\ ${ }^{2}$ Guangxi Shangsi Hualin Forest Industry Co., Ltd, Shangsi, Guangxi, 535500, China
}

\begin{abstract}
Based on the reflection, absorption and multiple reflection attenuation principle of composite shielding, composite fiberboard with multiple electromagnetic shielding functions was developed according to the structure design, which was made by filling with mineral powder and stainless steel nets, and then sprayed single face with conductive paint. The results show that: electromagnetic shielding effectiveness of the product is above $60 \mathrm{~dB}$ and reaches the better grade in $18.85 \mathrm{MHz}-1.46 \mathrm{GHz}$; the product filled with magnetite powder has better comprehensive mechanical properties than the product filled with barite powder, and its modulus of rupture, modulus of elasticity, internal bonding strength and thickness swelling rate of water absorption all reach the Chinese national standards.
\end{abstract}

Keywords: Electromagnetic shielding, mineral powder, stainless steel net, conductive paint, composite fiberboard.

\section{INTRODUCTION}

With the effects of electromagnetic radiation and electromagnetic interference for people's health and society increasing, it was very imperative to study new electromagnetic shielding function materials, and wooden electromagnetic shielding materials was one hot point of the study. Xianquan Zhang and Yixing Liu [1] had studied the ratio of stainless steel fiber which had affection on the electromagnetic shielding effectiveness of steel/wood composite fiberboard, and the results showed that: when the steel/wood composite fiber was on the bilateral surface layer of fiberboard with the proportion of 1:3, the electromagnetic shielding effectiveness could reach above $55 \mathrm{~dB}$ in $0-1.5 \mathrm{GHz}$. Xianquan Zhang and Yixing Liu [2] researched on the property of wood fiber-copper wire net composite MDF, and discovered that the effectiveness could reach above $60 \mathrm{~dB}$ in $9 \mathrm{kHz}-1.5 \mathrm{GHz}$ when copper wire net whose mesh was more than 60 was placed on surface layer. Xiansen Liu and Feng Fu [3] made three-layer compound larch plywood by adding respectively brass fiber and stainless steel fiber to urea-formaldehyde resin as conductive unit, and the effectiveness was in the range of $6.34 \mathrm{~dB}$ to $28.76 \mathrm{~dB}$ when added with brass fiber and $13.63-21.14 \mathrm{~dB}$ when added with stainless steel fiber in $9 \mathrm{kHz}-1.5 \mathrm{GHz}$. Xiansen Liu and Feng $\mathrm{Fu}$ [4] made three-layer compound Larch plywood by adding respectively super fine copper powder, super fine nickel powder and super fine graphite powder to urea-formaldehyde resin as conductive unit, the effectiveness was respectively $0 \mathrm{~dB}, 0.00$ $10.10 \mathrm{~dB}, 5.28-13.13 \mathrm{~dB}$ in $9 \mathrm{kHz}-1.5 \mathrm{GHz}$. Jintian Huang and Guangjie Zhao [5] had studied the electromagnetic shielding effectiveness of veneer plated with $\mathrm{Ni}$ by chemical method and discovered that the effectiveness could reach $30-60 \mathrm{~dB}$ in $0 \mathrm{~Hz}-$ 1.5GHz. Qiutie Zhi [6] made wooden electromagnetic shielding

*Address correspondence to this author at the Forestry College of Guangxi University, No.100, Daxue East Road; Nanning, Guangxi, P.R. China; Tel: +8613978177625, +8607713271438; Fax: +8607713271418; E-mail: glscw58@163.com materials by magnetron sputtering and discovered that the effectiveness could reach above $30 \mathrm{~dB}$ in $30 \mathrm{~Hz}-1.5 \mathrm{GHz}$. Keyang $\mathrm{Lu}$ and Feng $\mathrm{Fu}$ [7] had researched the property of electromagnetic shielding plywood laminated with conductive membrane; and discovered that when copper fiber was $200 \mathrm{~g} / \mathrm{m}^{2}$, the effectiveness was $39.30-61.75 \mathrm{~dB}$ and the average was $49.65 \mathrm{~dB}$ in $9 \mathrm{kHz}-1.5 \mathrm{GHz}$. Jiaqi Zhu et al. [8] had researched the composite of metal mesh and wood veneer, the results showed that the effectiveness could reach above $40 \mathrm{~dB}$ in $1 \mathrm{MHz}-1 \mathrm{GHz}$. Junling wang and Hongsha Guo et al. [9] have studied on wood-based panel whose surface was separately pasted with Ni-plated fabric and coarsening copper; the effectiveness of the wood-based panel whose surface was pasted with Ni-plated fabric was about $60 \mathrm{~dB}$ and the other one was above $70 \mathrm{~dB}$. Effectiveness grades was divided into five grades by Chohachiro $\mathrm{N}$ [10-12] in frequency of $50 \mathrm{MHz}-1 \mathrm{GHz}$ : (1) under 10dB: no effectiveness; (2)10-30dB: bad; (330-60dB: medium; (4)6090dB: good; (5)above 90dB: excellent.

In summary, currently, low effectiveness is seen in most of the current wooden electromagnetic shielding materials, and the better materials are very expensive because of using precious metals. This study, aiming at making a new kind of electromagnetic shielding function composite fiberboard, uses inexpensive natural ore powder (barite powder, magnetite powder) and two layers 80 mesh stainless steel nets and plated with copper-silver conductive paint.

\section{TEXT MATERIALS AND METHODS}

\subsection{Test Materials}

Barite powder: purchased from Guilin Brothers Mining Investment Co., Ltd, natural, 2000 mesh; contains barium elements (heavy metals element); effective in absorbing some harmful rays, such as X-rays [13].

Magnetite powder: purchased from Hechi Jinchuan Mining Co., Ltd, natural, 400 mesh; magnetite has a certain shielding effectiveness in the microwave frequency band, 
which is one kind of magnetic medium, includes a lot of magnetic ions and has favorable magnetic permeability and dielectric constant [14].

Stainless steel nets: 80 mesh, easy to be sawed, having a good shielding effectiveness in preliminary tests.

Polyurethane adhesive: JFW one-component polyurethane adhesive, purchased from Guangzhou Jingfengwei material Co., Ltd.

Copper-Silver powder electromagnetic shielding paint: YS-801 plastic silver-copper conductive paint purchased from Dongguan city Yousheng technology Co., Ltd.

Wood fiber: they were offered by Guangxi Hualin Forest Industry Co., Ltd; glue consumption was $12 \%$; in good condition.

\subsection{Test Methods}

\subsubsection{Structural Design of Composite Electromagnetic Shielding Fiberboards}

Three kinds of principle of weakening electromagnetic wave [15] : first, reflection attenuation, the reflection on the surface which weakens electromagnetic waves; second, absorption attenuation, electromagnetic waves which aren't reflected on the surface, go into the shield, and are absorbed by material, then are weakened; third, electromagnetic waves are weakened by multiple reflections and absorption attenuation within the shield. Shielding effectiveness can be calculated as the following Eq. (1) [16].

$S E=R+A+B$

In the Eq. (1), $S E$ instead of electromagnetic shielding effectiveness; $R$ instead of reflection attenuation; $A$ instead of absorption attenuation; $B$ instead of multiple reflection attenuation within the shield (If $A>15 \mathrm{~dB}$, it isn't meaningful). $A$ has nothing to do with the type of electromagnetic waves (electric or magnetic field), existing as long as electromagnetic waves through the shielding material; it is related to conductivity of the material, the magnetic permeability and material thickness. $R$ is not only related to the surface impedance of the material, but also related to the type of radiation and the distance between the radiation and the shield. $B$ mainly depends on the distribution of shielding material in the composite material.

Thus, conductive paint should be coated to the surface of composite fiberboards as a reflective layer in the test; the ore powder with absorbing properties was filled in composite fiberboards for the absorption attenuation; two stainless steel nets were separately placed close to the two surfaces of a composite fiberboard in order to achieve multiple composite shielding, shielding electromagnetic waves furthest. The shielding principle could be seen in Fig. (1).

\subsubsection{Hot-Pressing Process}

This study set plate thickness as $9 \mathrm{~mm}$, material density as $760 \mathrm{~kg} / \mathrm{m}^{3}$, pressing time as $10 \mathrm{~min}$, hot-pressing temperature as $170^{\circ} \mathrm{C}$, top hot-pressing pressure as $2.5 \mathrm{Mpa}$; and took a three-paragraph vacuum hot-pressing process.

Composite method: a net was used to make ore powder weighed at a quantitative proportion dispersed evenly to the fiber prepared in advance; stainless steel nets coated with polyurethane adhesive were separately placed in the two surfaces of the slab that would be pressed into a board; then conductive paint was coated to the surface of composite fiberboards. Among them, a sprayer was used for sizing evenly.

\subsection{Electromagnetic Shielding Effectiveness (SE) Testing}

According to the criterion "SJ20524-1995 Measurement of Shielding Effectiveness of Materials", composite fiberboards keep cooling $24 \mathrm{~h}$, then should be made out the standard disc specimens of diameter $115^{0}{ }_{-0.5} \mathrm{~mm}$; electromagnetic shielding effectiveness of the composite fiberboards would be tested by using DN15115 type of vertical flange coaxial test device (developed by Southeast University), and took the average value of the three specimens. The testing was entrusted to Research Institute of Wood Industry of Chinese Academy of Forestry to finish.

\subsection{Physical and Mechanical Properties Testing}

Modulus of rupture $(M O R)$, modulus of elasticity $(M O E)$, internal bonding strength $(I B)$ and thickness swelling rate of

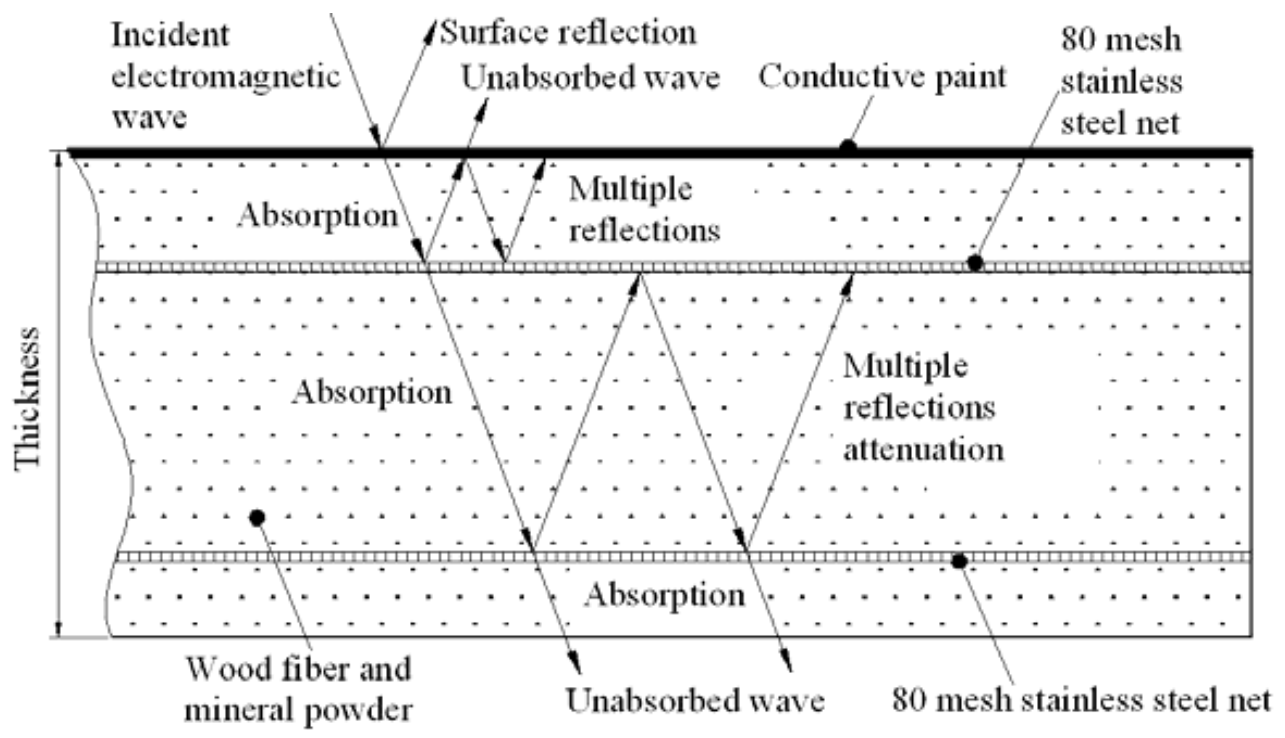

Fig. (1). Section structure and shielding mechanism of composite fiberboards. 
water absorption (TS) were tested according to China National MDF standard.

\section{RESULTS AND ANALYSIS}

\subsection{Analysis of Electromagnetic Shielding Effectiveness of Composite Fiberboard}

\subsubsection{Electromagnetic Shielding Effectiveness of Composite Fiberboards Made by Filling with Only Ore Powder}

Fig. (2) shows that, composite fiberboards made by filling with only ore powder had some electromagnetic shielding effectiveness which could reach $20 \mathrm{~dB}$ in the maximum in low frequency. Barite powder is better than magnetite powder. Barite powder, containing a lot of heavy metals elements of barium which can absorb rays in some frequency band, has a certain shielding effectiveness; magnetite powder has favorable magnetic permeability and dielectric constant, which also has a certain shielding effectiveness due to that the electromagnetic dipole and plays a role with electromagnetic waves, then absorbs some of the electromagnetic waves. The effect of barite powder was absorption attenuation and the main effect of magnetite powder was also absorption attenuation, but some reflection attenuation.

Whether the distribution of mineral powder in composite fiberboards is homogeneous or not had great influence on overall shielding and mechanical property. Its distribution in the section is shown in Fig. (3). Barite powder is uniformly distributed, which has a continuous distribution in the section. As magnetite powder and wood fiber surface have a large difference in the surface, it was difficult to mix them together homogeneously, and it would cluster and lead to uneven distribution.

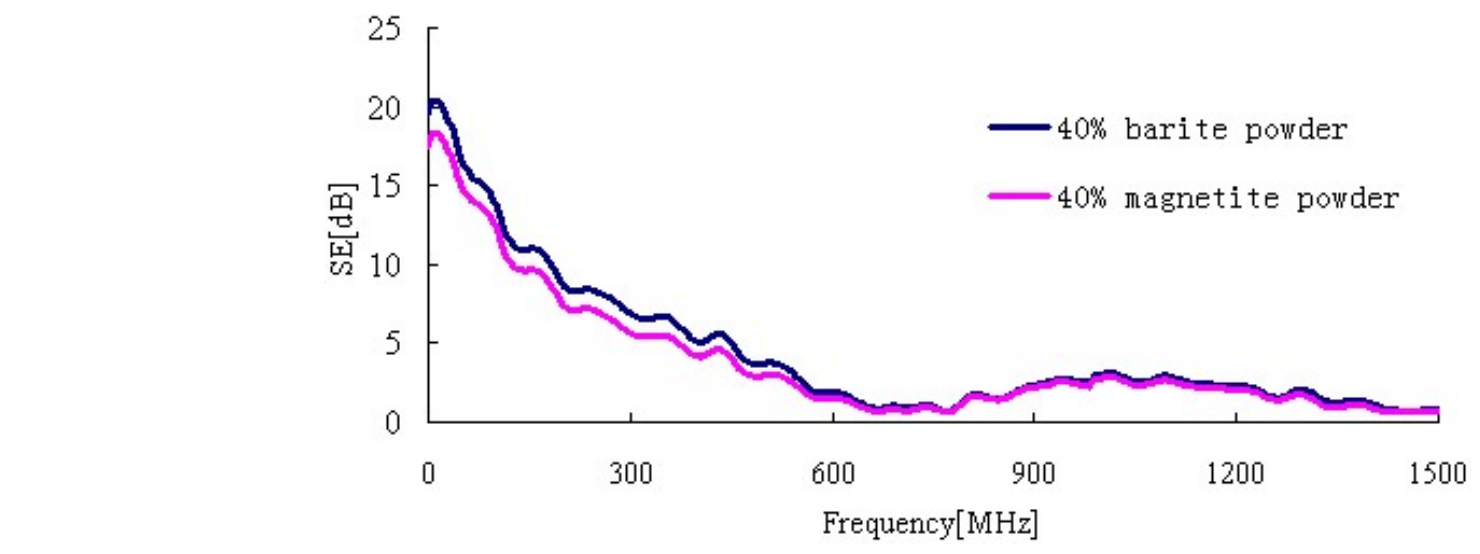

3.1.2. Electromagnetic Shielding Effectiveness of Composite Fiberboards Made by Filling Alone Stainless Steel Net and Spraying Conductive Coating

Fig. (4) shows that, the electromagnetic shielding effectiveness of the composite fiberboard using 80 mesh stainless steel nets is superior to the composite fiberboard made by spraying silver-copper conductive coating in the whole frequency band. That's to say, although the monolayer silvercopper coating has high conductivity, which primarily has the function of reflection, the shielding effectiveness is limited.

However, the net also primarily has the function of reflection, but when set two-layer nets separately in the board multiple reflection attenuation will be formed, so the shielding effectiveness is relatively high. Then, it could be summarized that multiple reflection structure has the excellent shielding function. But both of them have relatively low shielding effectiveness in the high frequency band and couldn't reach the requirements of $60 \mathrm{~dB}$.

Stainless steel nets are formed by many micro circuitries. When external electromagnetic wave are shot to the surface of stainless steel nets, it would inevitably cause magnetic flux change when passing through each micro circuitry, and then produce induced current and inductive magnetic field in circuits which could prevent the magnetic flux from changing in the circuit surface, and thereby shield external magnetic field. According to Faraday's law of electromagnetic induction, the reversed magnetic field produced by the induced current in circuits could offset external magnetic flux [17]. The electrical conductivity, magnetic permeability, mesh, wire diameter and contact resistance of the metal net all have an impact on its electromagnetic shielding effectiveness.

Fig. (2). SE of composite fiberboards filled with two mineral powders.

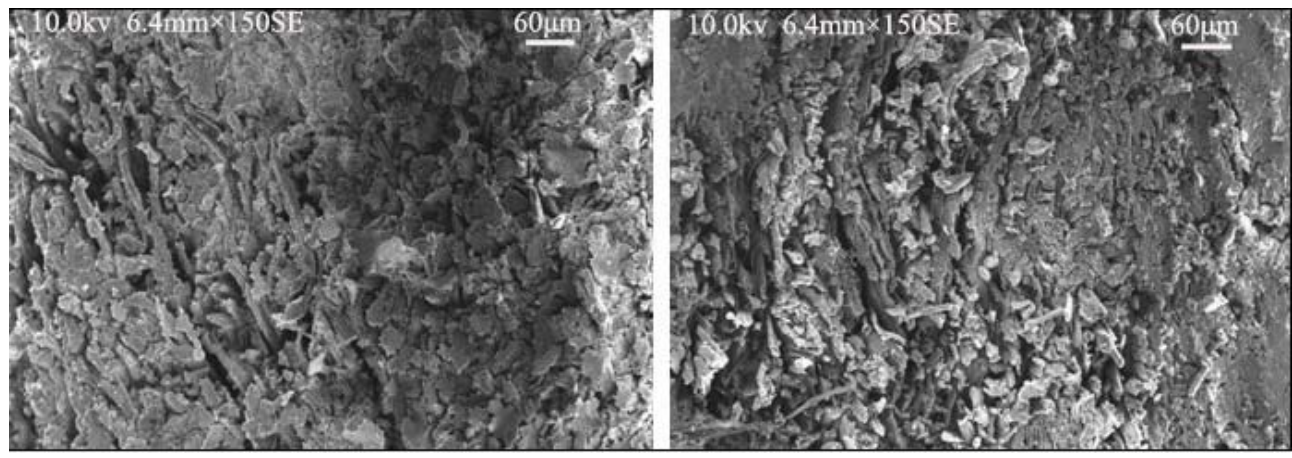

Fig. (3). The distribution of barite powder (left) and barite powder (right) in composite fiberboard. 


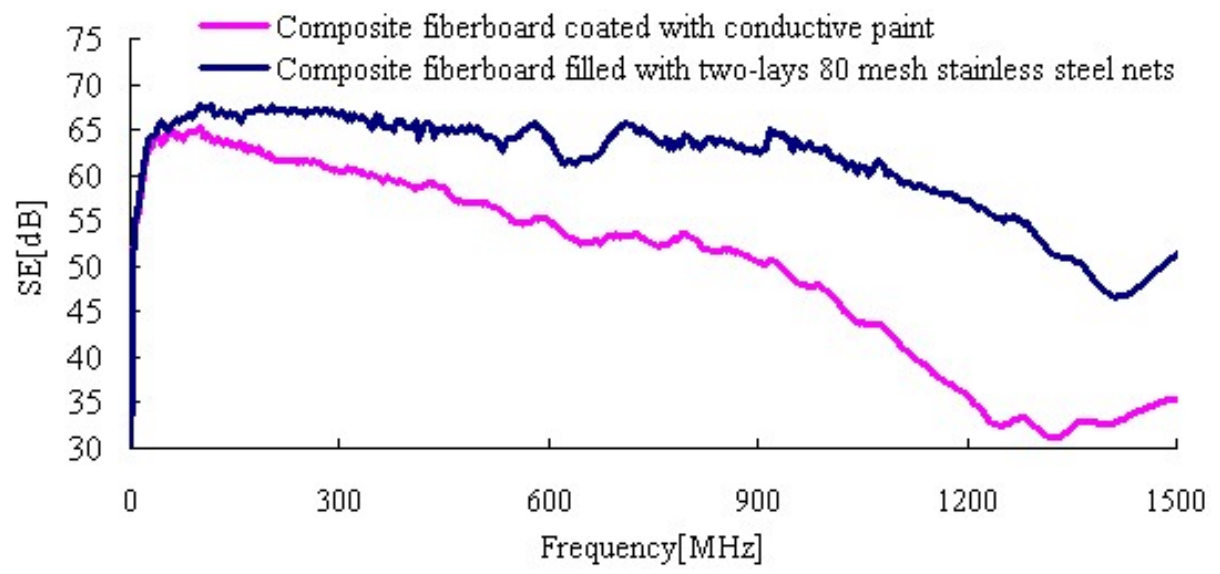

Fig. (4). SE of composite fiberboards filled with stainless steel nets and spraying conductive coating separately.

Although silver copper is good in electrical conductivity, as there are many contact points between powder and powder in the coating and powder and would also have a small amount of coating or additives package which would increase the contact resistance and reduce the electrical conductivity of the coating, so cause the decrease of electromagnetic shielding effectiveness. The result of element mapping and EDX analysis of paint coating are shown in Figs. $(\mathbf{5}, \mathbf{6})$.

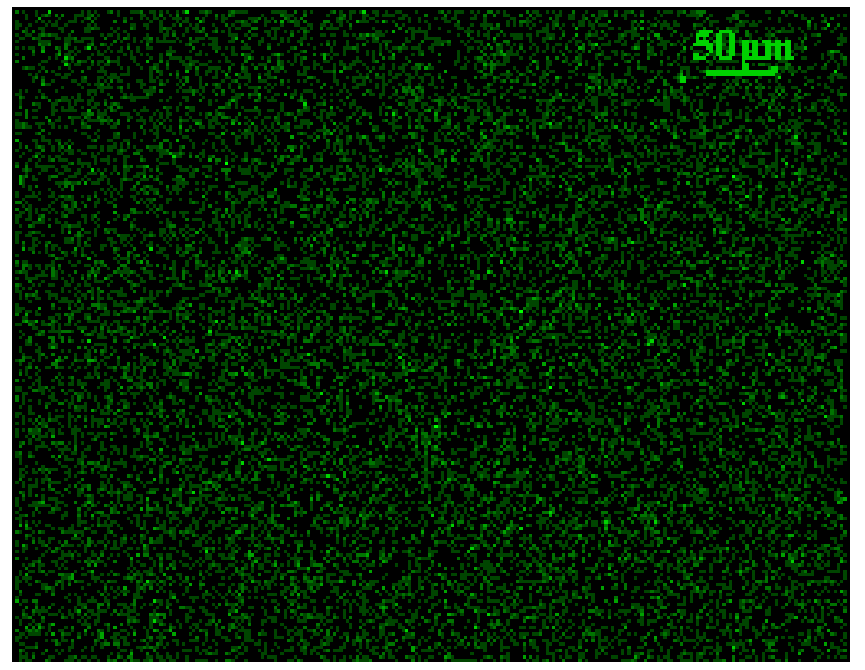

Fig. (5). Copper (green particles) element mapping of conductive paint coating.

The results show that: copper powder which has highest weight percentage is evenly distributed in coating; also, copper particles (green particles) contact mutually to form conductive net, so the coating has good conductivity.

\subsubsection{Electromagnetic Shielding Effectiveness of Composite Fiberboards}

Fig. (7) shows that, after compounding three materials, electromagnetic shielding efficiency of the functional fiberboards in the range of $50 \mathrm{MHz}$ to $1000 \mathrm{MHz}$ is more than $60 \mathrm{~dB}$, which achieve better performance level; among them, shielding bandwidth of more than $60 \mathrm{~dB}$ of composite fiberboards filled with barite powder is in the range of $18.85 \mathrm{MHz}$ to $1.5 \mathrm{GHz}$, and when filled with magnetite powder that is in the range of $18.85 \mathrm{MHz}$ to $1.46 \mathrm{GHz}$. So, barite has slightly better effectiveness than magnetite in the high frequency, because barite containing barium elements has strong absorption attenuation function which could effectively absorb the electromagnetic wave of high frequency.

The results form Fig. (7) shows that, the attenuation shielding method which incorporates reflection, absorption and multiple reflections have a very good shielding effectiveness. Its mechanism should be as follow: when electromagnetic wave is incident on the composite fiberboard surface, its surface electromagnetic shielding coating reflects a part of electromagnetic waves; the part that hasn't been reflected will continue to go into composite fiberboard inner layer, then reach to surface of one of stainless steel nets; stainless steel net produces an opposite magnetic field, which should make part of the electromagnetic wave reflect; and then the reflection of the electromagnetic waves incident to the surface coating, also continue to multiple reflections; at the same time, mineral powder filled dispersedly in the composite fiberboard, constantly absorb the electromagnetic waves which reflected many times. Electromagnetic waves through the first layer stainless steel net, would continue to be incident on the second stainless steel net and reflect partly; as a result of two layer stainless steel nets placed in two surface, existing a certain spacing between two nets, the chance for scattered mineral powder to absorb the electromagnetic wave which happen multiple reflection are increasing; finally complete the whole process of composite shielding.

\subsection{The Physical and Mechanical Properties of Composite Fiberboards}

According to Chinese national medium density fiberboards standard GB/T 11718-2009, the physical and mechanical performance of fiberboard are that $M O R$ is more than $23 \mathrm{MPa}$, and $M O E$ is more than $2700 \mathrm{MPa}$, and $I B$ is more than $0.6 \mathrm{MPa}$, and $T S$ is less than $20 \%$.

The physical and mechanical performance test results of developed composite fiberboards are showed in Table 1. All the data are the average of six times test results.

It can be known from Table 1, when fiber sizing quantity achieved $16 \%, M O R, M O E, I B, T S$ of composite fiberboards added with $30 \%$ magnetite powder and filled with two-layers 80 mesh stainless steel nets have basically reached national standard requirements. $M O R, M O E$ of composite fiberboards 


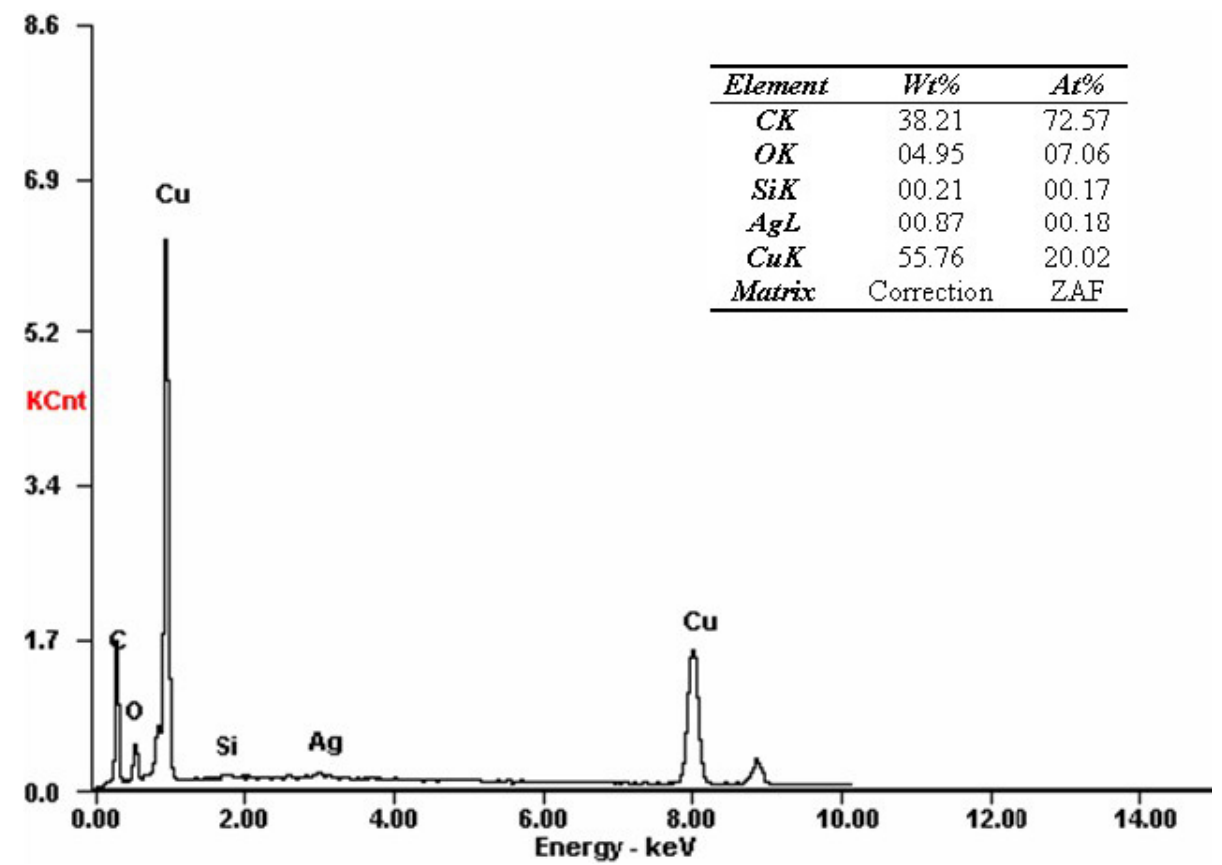

Fig. (6). EDX analysis of conductive paint coating (ZAF, Zero Alignment Feature).

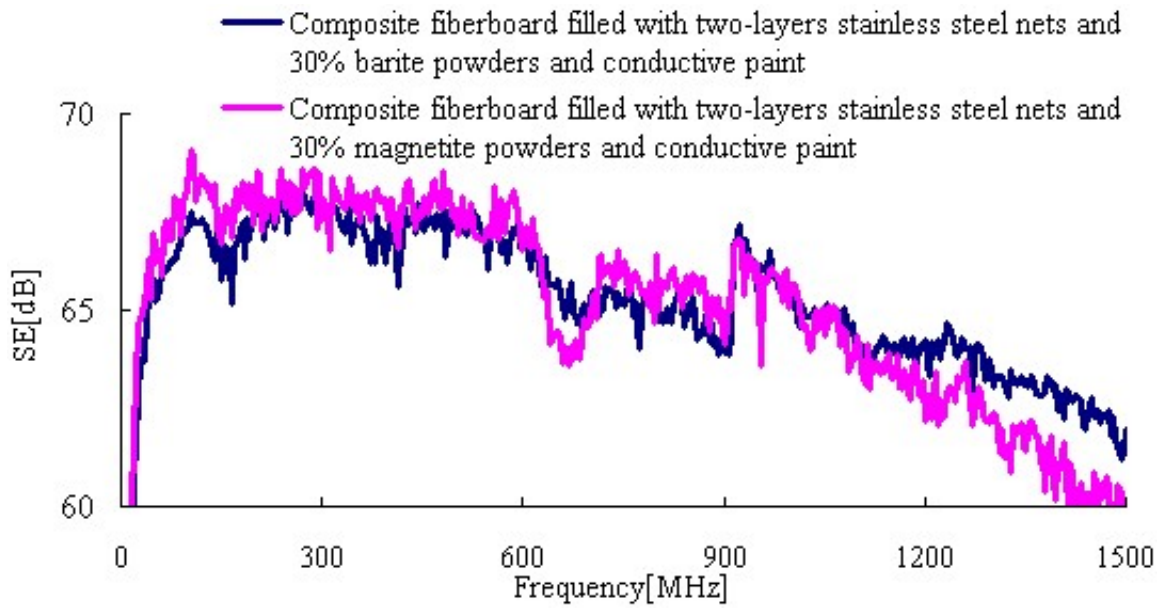

Fig. (7). SE of the composite fiberboards.

added with $30 \%$ barite powder and filled with two-layers 80 mesh stainless steel nets have all reached national standard requirements, but $I B$ and $T S$ haven't reached national standard requirements. The reason maybe that chemical stability of barite powder is good, and thermal conductivity is bad, and the density is relatively small; then the filling amount was more, which decreases the opportunity for mutual agglutination between each wooden fiber, then affects the TS property; also the barite powder has better hydrophilicity and higher $T S$.

\section{CONCLUSIONS}

(1) When the composite fiberboard was filled with the mixture of mineral powder and stainless steel nets,

Table 1. The Physical and Mechanical Properties of Composite Fiberboards

\begin{tabular}{|c|c|c|c|c|c|c|}
\hline Types of Composite Fiberboards & Sizing Quantity [\%] & $\begin{array}{l}\text { Data } \\
\text { Type }\end{array}$ & $\operatorname{MOR}[\mathrm{MPa}]$ & MOE [MPa] & $I B$ [MPa] & $T S[\%]$ \\
\hline $\begin{array}{l}\text { Composite fiberboard filled with } 30 \% \text { barite powder+ } \\
\text { two-layers } 80 \text { mesh stainless steel nets }\end{array}$ & & $\begin{array}{l}\text { Avg. } \\
\text { CV }\end{array}$ & $\begin{array}{l}36.59 \pm 1.71 \\
4.7\end{array}$ & $\begin{array}{l}5654.09 \pm 280.09 \\
5.0\end{array}$ & $\begin{array}{c}0.38 \pm 0.06 \\
15.8\end{array}$ & $\begin{array}{c}25.7 \pm 2.3 \\
8.9\end{array}$ \\
\hline $\begin{array}{l}\text { Composite fiberboard filled with } 30 \% \text { magnetite } \\
\text { powder+ two-layer } 80 \text { mesh stainless steel nets }\end{array}$ & & $\begin{array}{l}\text { Avg. } \\
\text { CV }\end{array}$ & $\begin{array}{c}30.32 \pm 2.92 \\
9.6\end{array}$ & $\begin{array}{l}4761.03 \pm 323.23 \\
6.8\end{array}$ & $\begin{array}{l}0.67 \pm 0.05 \\
7.5\end{array}$ & $\begin{array}{c}19.5 \pm 0.38 \\
1.9\end{array}$ \\
\hline
\end{tabular}

$M O R$, modulus of rupture; $M O E$, modulus of elasticity; $I B$, internal bonding strength; $T S$, thickness swelling rate of water absorption. 
and sprayed with conductive coating on its surface, its electromagnetic shielding effectiveness in the frequency band of $50-1000 \mathrm{MHz}$ was respectively up to $60 \mathrm{~dB}$, which have achieved a good level; among them, the shielding bandwidth above $60 \mathrm{~dB}$ of the composite fiberboard filled with barite powder is $18.85 \mathrm{MHz}-1.5 \mathrm{GHz}$, and the shielding bandwidth filled by magnetite powder is $18.85 \mathrm{MHz}-1.46 \mathrm{GHz}$.

(2) When fiber sizing quantity reaches $16 \%, M O R, M O E$, $I B, T S$ of the composite fiberboard, which was filled with the mixture of magnetite powder of $30 \%$ and two-layer stainless steel nets of 80 mesh together, have basically reached Chinese national standard requirements. The $M O R$ and $M O E$ of the composite fiberboard, which was filled with the mixture of barite powder of $30 \%$ and two-layer stainless steel nets of 80 mesh together, have reached national standard requirements, while its $I B$ and $T S$ haven't and need further study.

(3) It is a great help to improve the electromagnetic shielding effectiveness of the composites by conducting the reasonable structure design according to the composite shielding mechanism of reflection, absorb and multiple attenuation.

\section{ACKNOWLEDGEMENTS}

This work was partially supported by Guangxi Provincial Scientific Research Foundation [NO: 10100022-26] and Guangxi Provincial Forestry scientific research Foundation [NO: 2009-7] and National Forestry Public Welfare Industry Scientific Research Special Project [NO: 201004070].The authors are grateful to the Forestry Agency of China and scientific Agency of Guangxi Province for its financial support for this work.

\section{CONFLICT OF INTEREST}

Declared none.

\section{REFERENCES}

[1] Zhang X, Liu Y. Study on properties of steel/wood fiber composite MDF. China Wood Ind 2005; 19(2): 12-6.

[2] Zhang X, Liu Y. Study on the wood fiber-copper wire net composite MDF. China For Prod Ind 2004; 31(5): 15-9.

[3] Liu X, Fu F. Composite of metal fiber and wood veneer. For Machinery Woodworking Equip China 2008; 36(11): 27-30.

[4] Liu X, Fu F. Study on Performance of electro- conductive powder and veneer composite. J Nanjing For Univ (Nat Sci Ed) China 2009; 33(2): 95-8.

[5] Huang J, Zhao G. Electroconductivity and electromagneticshielding effectiveness of nickel- plated veneer. China For Prod Ind 2006; 33(1): 14-7.

[6] Qiu T. The research of electromagnetic shielding materials made of wood. Master Thesis. China: Chinese Academy of Forestry Sciences 2008

[7] Lu K, Fu F, Cai Z, et al. Study of properties of electromagnetic shielding plywood laminated with conductive sheets. J Build Mater China 2011; 14(2): 207-11.

[8] Zhu J, Luo Z, Huang Z. Composition of wire net and wood veneer. China Wood Ind 2001; 15(3): 5-7.

[9] Wang J, Guo H, Li Y, et al. Preparation and characteristics of surface-type electromagnetic shielding wood-based composites. China Wood Ind 2009; 23(1): 21-3.

[10] Ma Y, Luo Z. A review of wood/metal composite materials. Anhui Chem Ind China 2006; (3): 37-8.

[11] Chohachiro N, Yaomi K, Kei U, Shunichi S. Electromagnetic shielding particleboard with nickel-plate wood particle [J]. J Wood Sci Jpn 1989; 35 (12): 1092-9.

[12] Chohachiro N, Yaomi K, Kei U, Shunichi S. Electromagnetic shielding effectiveness of particleboard containing nickel-metalized wood-particle in the core layer. J Jpn Wood Res Soc 1990; 36(7): 531-7.

[13] He D, Dong F, Deng Y. Application of functional minerals in cement sludges capable of preventing radon and radiation. Mutilpurpose Util Miner Resour China 2005; (2): 18-20.

[14] Guan D, Wang S. The electromagnetic wave-absorbing properties of mineral materials and their application. Mutilpurpose Util Miner Resour China 2006; (5): 17-20.

[15] Wang J. Mechanism and development of electromagnetic shielding materials. New Chem Mater China 2002; 30(7): 16-8.

[16] Kaiser BE, The principles of electromagnetic compatibility. Beijing: Publishing House of Electronics Industry 1985.

[17] Wan P. Primary exploration of electromagnetic shielding problems of metal nets. Safety EMC China 1999; (3): 27-9. 